University of New Hampshire

University of New Hampshire Scholars' Repository

$10-1988$

\title{
Modifications and Improvements to the Sea Beam System on Board R/V Thomas Washington
}

Christian de Moustier

University of California - San Diego

T Hylas

Scripps Institution of Oceanography

J C. Phillips

Scripps Institution of Oceanography

Follow this and additional works at: https://scholars.unh.edu/ccom

Part of the Oceanography and Atmospheric Sciences and Meteorology Commons

\section{Recommended Citation}

de Moustier, Christian; Hylas, T; and Phillips, J C., "Modifications and Improvements to the Sea Beam System on Board R/V Thomas Washington" (1988). IEEE Oceans. 767.

https://scholars.unh.edu/ccom/767

This Conference Proceeding is brought to you for free and open access by the Center for Coastal and Ocean Mapping at University of New Hampshire Scholars' Repository. It has been accepted for inclusion in Center for Coastal and Ocean Mapping by an authorized administrator of University of New Hampshire Scholars' Repository. For more information, please contact Scholarly.Communication@unh.edu. 


\title{
MODIFICATIONS AND IMPROVEMENTS TO THE SEA BEAM SYSTEM ON BOARD R/V THOMAS WASHINGTON
}

\author{
C. de Moustier, T. Hylas and J.C. Phillips \\ Scripps Institution of Oceanography \\ Shipboard Computer Group, A-023 \\ La Jolla, California 92093
}

\begin{abstract}
A Sea Beam multibeam bathymetric survey system has been in operation on board the R/V T. Washington of the Scripps Institution of Oceanography since December 1981. In response to operational requirements, the engineers of the Shipboard Computer Group at Scripps have implemented a number of modifications to the system's Narrow Beam Echo-Sounder and to its Echo Processor. These include the design and construction of a digital pitch compensator, the ability to use a variety of sensors for vertical reference, the design and construction of hardware test equipment, of an interface to the shipboard DEC VAX-11/730 computer for data logging and for automation of start-up procedures as well as for performance monitoring. Some of the modifications have prompted the manufacturer of the Sea Beam system, General Instrument Corporation, to upgrade the system they have delivered in the past 4 years to match the corresponding improvements implemented at Scripps and which could prove useful to Sea Beam operations on other ships.
\end{abstract}

\section{INTRODUCTION}

A Sea Beam bathymetric survey system, manufactured by the General Instrument Corporation (GIC), was installed on board the $\mathrm{R} / \mathrm{V} \mathrm{T}$. Washington of the Scripps Institution of Oceanography (SIO) in the fall of 1981. This system is a multibeam echo-sounder designed to measure depth on 16 discrete beams, with $22 / 3^{\circ}$ angular resolution, and produce a swath of contours along the ship's track. It consists of two main components: a narrow beam echo-sounder and an echo processor (Fig. 1). The echo-sounder consists of two hullmounted transducer arrays, installed at right angle to each other in a $T$ configuration, their associated signal generator, pitch and roll compensators, timing unit, power amplifiers, preamplifiers, and beam. forming network. The echo-processor utilizes a Data General Eclipse S-130 real-time computer for the digitization of the echo signals received and detected on each beam, for bottom detection and tracking, for geometric correction (roll and refraction), and for computing depths and horizontal distances to be displayed as an instantaneous depth profile or contoured and output to a swath plotter. More extensive descriptions of the Sea Beam system and its operation are found in Renard and Allenou [1], Farr [2] and de Moustier and Kleinrock [3].

Following acceptance tests and sea trials in December of 1981 aboard the R/V T. Washington, the system has been in operation an average of $\mathbf{1 8 0}$ days per year over the past seven years, with relatively little down time due to system failure. Several failures of the pitch compensation unit occurred in the first two years of operation.
During this time, the engineers and the technicians of SIO's Shipboard Computer Group (SCG), who operate and maintain the system, began to modify some of the components of the system to improve reliability and performance as well as to facilitate the maintenance tasks. In the last four years, the improvements to the operation and maintenance of the Sea Beam system have been tied to an upgrade of the shipboard computer from an IBM 1800 to a DEC VAX-11/730 system, henceforth referred to as VAX.

In the following we discuss the design philosophy adopted for logging the bathymetric data output by the Sea Beam system on the VAX, and its implications for expansion with new hardware or additional interfaces. We then describe the implementation of the new

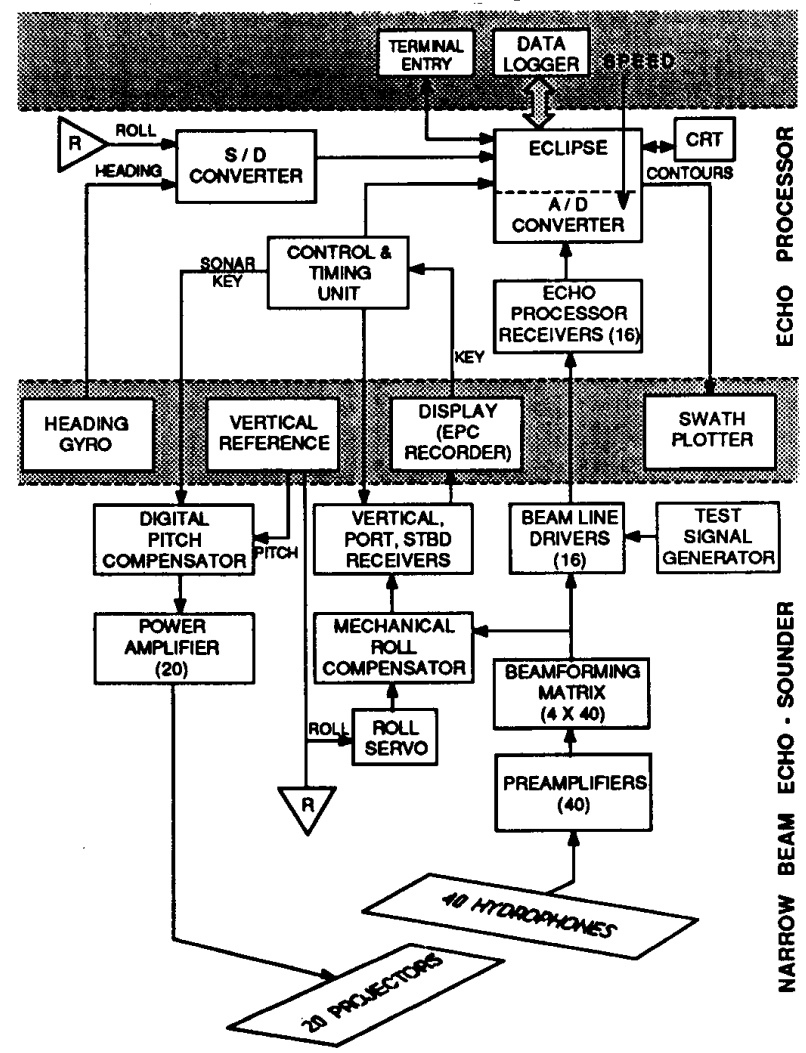

Figure 1. Block diagram of the Sea Beam system with its two main sub-systems: a Narrow Beam Eacho Sounder and an Echo Processor. Elements in the shaded areas are peripherals and external sensors. 
components designed by SCG which include a digital pitch compensator, a sound source synchronizer and test equipment for system calibration. Finally, we discuss the modifications that have improved the reliability of the system and facilitated the operator's tasks such as an automated parameter initialization procedure and enhanced output capabilities, the ability to use various vertical reference sensors, and a capability to record the acoustic waveforms received on each of the 16 preformed beams.

\section{DATA LOGGING AND PROCESSING}

During each transmission cycle, the Echo Processor in the Sea Beam system computes depths and cross-track distances from the seafloor echoes received on each of the 16 preformed beams. These data are displayed on an oscilloscope as an instantaneous depth profile across track, as well as on a swath plotter as a contour chart. In addition the data are sent to a data logging system. In this section. we describe the current hardware which conveys the data from the Echo Processor to the Data Logger aboard the R/V T. Washington.

\section{Sea Beam to VAX Interface via STD-GPIB Bus}

The data logging option sold by GIC only consists of two magnetic tape drives. An additional computer is required to merge the bathymetric data output by the Sea Beam system with the ship's navigation data in order to produce a contour map in geographic coordinates. As the R/V T. Washington was already equipped with an IBM 1800 real-time computer configured to log and process the ship's navigation and underway geophysical data (i.e. magnetics, gravity), SIO opted to use this computer to $\log$ and process Sea Beam data as well. In February 1984, the IBM 1800 was replaced by a DEC VAX-11/730 computer system [4].

The front end of the VAX system has microprocessor controlled input/output devices utilizing the IEEE 961 Standard (STD) bus protocol in conjunction with an IEEE 488 General Purpose Interface Bus (GPIB) bus controller (Fig. 2). The STD bus is an 8 bit data bus controlled by a Z80 microprocessor. Data from a variety of sources are transferred over this bus in an 8-bit parallel format under control of the microprocessor and stored in local memory until interrogated by a GPIB controller in the VAX. The controller subsequently sends these data to disk storage through a separate STD to GPIB interface. In this manner the VAX is free to perform higher priority tasks before polling the individual STD bus devices for their data [4], [5].

The choice of the STD bus architecture to route Sea Beam data to the VAX was prompted by a larger real-time data acquisition scheme involving several other underway geophysical measurements (e.g. gravity, magnetics, single channel seismics) as well as the ship's navigation (e.g. Loran C, Transit Satellites, dual-axis Doppler speed log) [6]. This architecture has the advantage of providing a versatile instrument interface and data buffering system independent of the main computer [4]. It also allows the addition of new sensors without affecting the performance of the main computer, and the choice of sensors is not limited to those which have internal data buffering capability. By comparison, computers installed by the NECOR group in support of Sea Beam operations, and tasked only with acquisition and processing of Sea Beam data and the ship's navigation data, rely on sensors providing a serial RS-232C output [7].

The Sea Beam STD interface uses line receivers and differential drivers on its data lines to ensure a high immunity to noise for the data flow. The data flow between Sea Beam and the Data Logger is illustrated in Figure 2.

Every transmission cycle, the Data Logger output board in Sea

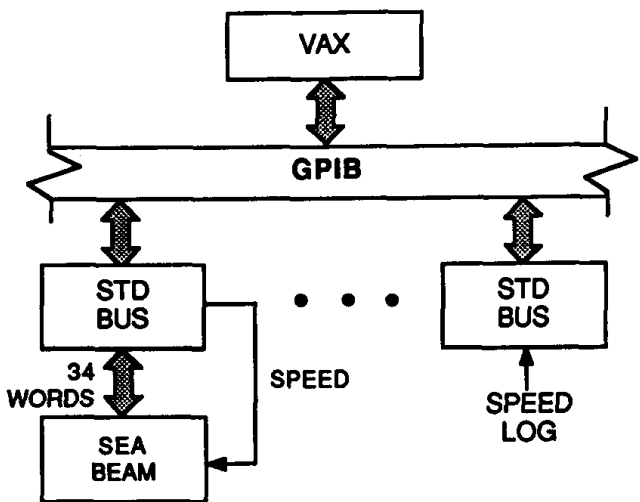

Figure 2. Data flow from Sea Beam to the VAX via the STD-GPIB buses.

Beam's Echo-Processor sends 34 words (i.e. up to 16 depths and 16 horizontal distances as well as time in minutes within the hour) and ships heading in a bit parallel, word serial format. That is, a 16 bit word is transmitted in parallel and all 34 words are transmitted serially. Three handshake signals associated with the Data Logger output board ensure the proper synchronization of the data to the Sea Beam STD Interface board.

Another function of the Sea Beam STD interface is to output the ship's speed to Sea Beam. This is done by sending a digital representation of the ship's speed to the STD bus from the VAX via GPIB. This information is then changed into the proper analog voltage by a digital-to-analog converter and sent to Sea Beam for use in the real time swath chart record. The rest of the hardware on this interface is used for address decoding and timing needed for communication with the Z80 microprocessor.

\section{NEW DESIGNS}

The Z80 microprocessor architecture described in Section II has been used in the design of two new components discussed in this section: a pitch compensator and multiple sound source synchronizer. Retaining the same microprocessor architecture had the advantage of simplifying maintenance, as spare parts would be interchangeable between the various data acquisition systems and the new components, and of capitalizing on the programming expertise already acquired for the $\mathrm{Z} 80$ microprocessor.

\section{A Microprocessor Controlled Pitch Compensator}

The transmitter array of the Sea Beam system consists of 20 projectors mounted along the ship's keel. The array is electronically steered to compensate for the pitching of the ship. To accomplish this, a pitch compensator alters the phase of the input signal fed to each projector relative to the center of the array, projector \#10. This method ensures that the maximum response axis of the transmitted radiation pattem remains aligned with true vertical.

The system originally installed on the R/V T. Washington used a mechanical pitch compensator in which a synchro signal representing the ship's pitch angle was fed to a control transformer driving a servo motor in a one speed closed servo loop. The servo motor was mechanically coupled to a gear train which was connected to 20 resolvers. Each resolver was geared so that when a pitch synchro signal was input, the resolver tumed accordingly and output the proper phase adjusted signal to each of the twenty projectors. According to GIC, very few problems had been reported on similar units installed in other ships equipped with a Sea Beam system or 
only with a narrow beam echo-sounder. However, many hours of maintenance and down time were incurred because of failure of the pitch compensator on the R/V T. Washington. The gear train connected to each resolver would easily slip and bind causing failure of the compensator or, far worse, subtle inaccuracies of the output signals. In addition, the device's small physical dimensions ( $12 \times 3 \times 4$ inches) made it difficult to repair or adjust, particularly in a seagoing environment. For this reason SCG decided to design and build a digital pitch compensator that would perform the same task without the problems associated with a mechanical design.

The design had to meet the same input-output criteria as the mechanical compensator did. To accomplish this we use an electronically controlled system based on a Z80 microprocessor and the STD bus. As shown in Figure 3, the resulting design requires six circuit boards:

(1) a Central Processor Unit board which contains the Z80 microprocessor and associated hardware used to control the operation of the system. It also contains memory devices for permanent and temporary storage of information.

(2) a Synchro to Digital Converter board which converts the pitch signal supplied by the vertical reference (Fig. 1) from synchro to digital format. This information is then used by the processor to output angle and counter delay values.

(3) a Display board which outputs pitch angle obtained from the processor to a LED display on the front panel of the compensator drawer.

$(4,5)$ two counter boards accepting delay values from the processor as input to 20 counter circuits which output a $12,158 \mathrm{~Hz}$ square wave for each of the 20 projectors. One counter board contains counters for channels 1-10 the other contains counters for channels 11-20. In addition one of the counter boards has the necessary circuitry required to synthesize a $12,158 \mathrm{~Hz}$ square wave.

And (6), a filter board which changes the square waves output by the 20 counters to sine waves required to drive the transmitter shaper amplifiers.

The system is run by software installed in permanent memory on the CPU board. This software is divided into three sections: a control program, a counter delay table and an angle display table. The function of the software is to wait for a sonar key from Sea Beam's timing logic and, upon receipt, read the pitch angle information from the synchro-to-digital (S/D) converter. This angle is then used to index a lookup table containing the appropriate phase delays to be loaded into each of the 20 counters on the counter boards. The software also uses a lookup table containing angle values to output a pitch angle to a LED display.

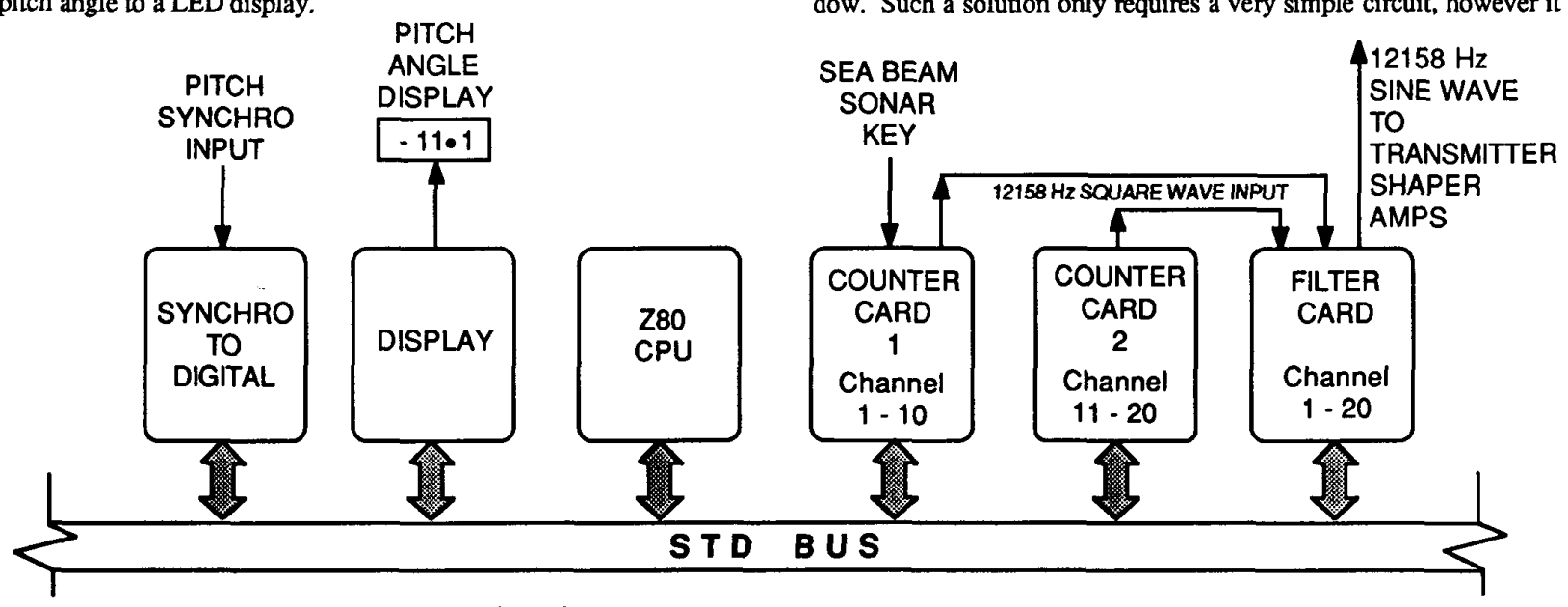

Figure 3. Architecture of SIO's digital pitch compensator.
Each counter is loaded by it's own LOAD command from the software to an initial count value. A $3.112448 \mathrm{MHz}$ clock signal is then gated on by a Modified Sonar Key (MSK) and they commence counting. The Modified Sonar Key is a signal created in software which limits the length of the pulse sent to the transmitter to $7 \mathrm{~ms}$ to prevent damage to the transmitter power amplifiers. The counters are wired as divide by eight so that by using the high order bit on each counter, a $12,158 \mathrm{~Hz}$ output is obtained. The resulting square wave is then gated with MSK and the signals at the output of the counter boards are passed to the filter board. The filter board is comprised of 20 switched capacitor filters which were selected for their stability and accuracy. The filters change the $12,158 \mathrm{~Hz}$ square wave output by the counter boards into a $4.5 \mathrm{v}$ p-p sine wave. This is done to remain compatible with the original system which requires a sine wave input to the transmitter shaper amplifiers. These amplifiers then output a signal to the transmitter power amplifiers, which send a $7 \mathrm{~ms}$ burst of $12,158 \mathrm{~Hz}$ energy to the projectors.

This digital pitch compensator was first tested on the R/V $r$. Washington in 1984, and became an integral part of the system in August 1985, greatly improving the Sea Beam system's reliability and decreasing system down time. The simple replacement of STD bus modules in the compensator has also decreased repair time on the system. In parallel with SCG's efforts, GIC also developed a digital pitch compensator to replace the mechanical unit. All the Sea Beam systems delivered by GIC since 1985 are equipped with a digital pitch compensator.

\section{Multiple Sound Source Synchronizer}

During geophysical surveys carried out with the R/V T. Washington, a $3.5 \mathrm{kHz}$ subbottom profiler, a one or two channel seismic profiler and the Sea Beam system are often operated simultaneously. This creates conflicting transmit-receive sequences and these systems interfere with each other. The Sea Beam system is affected the most by such interferences as its bottom tracking algorithm may lock on an interfering signal for a series of transmit cycles and generate erroneous bathymetry or artifacts in the contours which could be mistaken for a bottom feature [3]. Experience has shown that operator intervention to avoid interference, by slewing sound sources away from each other, usually happens after the fact. Moreover, over rough terrain the task becomes rather demanding when more than two sound sources are involved. When the Sea Beam system is operated in conjunction with the $3.5 \mathrm{kHz}$ subbottom profiler, interferences between the two systems can be avoided by simply gating out the $3.5 \mathrm{kHz}$ transmission during a Sea Beam reception window. Such a solution only requires a very simple circuit, however it 
is inadequate whenever a third sound source is also operated simultaneously.

To deal with this problem, SCG designed and built a multiple sound source synchronization box, also know as "Synch Box", which schedules the firing rates of the three sound sources according to selectable priority assignments and to timing parameters computed by a Fortran program running on the VAX. As this system is described in detail by Phillips et al [8], only a brief overview of its components is given here.

The hardware of the Synch Box uses the same Z80 microprocessor and STD bus system described above, and it consists of three circuit boards (Fig. 4). (1) a CPU board containing the $\mathrm{Z80}$ microprocessor, EPROM firmware for the application program and RAM memory for system management functions, as well as an upgraded $4 \mathrm{MHz}$ crystal oscillator with an accuracy of 5 parts per million. (2) an input-output board which orchestrates the events associated with each sound source under control of the firmware. And (3) a display interface board which displays the most current center beam depth measured by the Sea Beam system on a 5-digit LED readout as well as transmit events for each of the sound sources on individual LED's. A continuous two-way communication takes places between the Synch Box and the VAX acting as a host. The firmware checks the status of the various sound source systems and relays the information to the scheduling algorithm running on the VAX. It also controls the triggering of source and graphic recorder events by loading counters with the appropriate delay parameters calculated by the scheduling algorithm and passed by the VAX.

This system has been operational on the R/V T. Washington since the winter of 1987 . When all three sound sources are in operation, the seismic system, having the most rigid firing rate requirements, is given first priority, the Sea Beam system comes next and the $3.5 \mathrm{kHz}$ subbottom profiler is last. Once the initial parameters have been loaded and the scheduling program started, the Synch Box operation requires no further operator intervention.

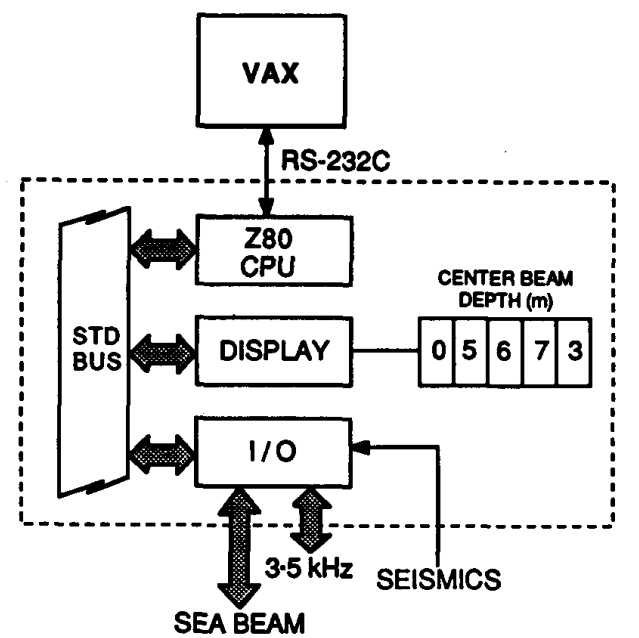

Figure 4. Architecture of SIO's multiple sound source synchronizer.

\section{Test Equipment}

To ensure the continued optimum performance of the Sea Beam system, a regular maintenance schedule must be observed, requiring test procedures and measurement equipment. In general, SCG's engineers use the test procedures recommended by GIC, however specific test equipment and procedures were developed in-house to facilitate the calibration of the transmitter power amplifiers and to measure the response of the Echo Processor receivers.

To calibrate the transmitter power amplifiers, the most vital piece of test equipment is a digital storage oscilloscope (DSO) with a built-in GPIB interface. A DSO is necessary to be able to verify the shape of the $7 \mathrm{~ms} \mathrm{CW}$ transmit pulse as well as to measure the phase difference between the outgoing voltage and the retum current in each power amplifier. Taking advantage of the DSO's ability to digitize, save a waveform and transfer it to a host computer, SCG developed a simple procedure to perform the calibration. The square wave output of each power amplifier is run through a $12 \mathrm{kHz}$ bandpass filter box built in house and digitized with the DSO. These data are then sent to the VAX through the GPIB/STD interfaces for analysis. Currently, the values of the 20 projector channels are entered into a Fortran program which calculates the output power corresponding to the measurement, and each channel is determined to be either in or out of specifications and adjusted accordingly. We intend to remove the filter box from this operation and use digital filtering programs on the VAX to perform the same function. This procedure proved far more reliable and accurate than one based on visual readings from an analog or non-storage oscilloscope.

SCG also built an echo delay generator to perform the necessary tests on the Echo Processor receivers. This device contains a $12,158 \mathrm{~Hz}$ oscillator, matching the transmit frequency of the Sea Beam system, and presetable counters and amplifiers. After terminating the hydrophone inputs with a resistor network, the delays and amplitudes specified by the manufacturer are set in the device, and the response of each receiver is checked for accuracy following the procedure recommended by GIC. This device has greatly simplified the method of insuring accurate response of the Echo Processor receivers.

\section{MODIFICATIONS}

The modifications described in this section have been implemented to (1) facilitate the task of the operator and reduce the potential for error by streamlining the Sea Beam start-up procedure, and by expanding the output capabilities of the system; (2) improve the system's reliability by being able to switch one of three vertical reference sensor online, and by alleviating a problem affecting the accuracy of the ship's roll information received by the EchoProcessor, and (3) give access to the acoustic signals at the output of the beamformer to allow their recording for further analysis.

\section{Downloading of Sea Beam Initialization Parameters}

The operator's console provided by GIC to communicate with the Eclipse computer in Sea Beam is a DECwriter printing terminal, serving both as keyboard for parameter entry and as printer for output of system messages by the Eclipse computer. On the R/V T. Washington, this console has been replaced by a direct RS-232C connection to the VAX. Console messages sent by the Eclipse computer are now saved in a $\log$ file on the VAX and echoed on a GraphOn CRT terminal with an attached Okidata printer for optional hard-copy output. A switch-box was added to allow this terminal to be used with either the Sea Beam Eclipse processor or the VAX host computer and serve as a laboratory checkpoint to monitor the status of a variety of equipment.

With this setup and a UNIX* C-shell script, it was relatively simple to automate the procedure required to initialize the Sea Beam system by downloading a parameter file from the VAX to the Eclipse computer. The parameters in this file include the Leg number, the date, the time of day, the ship's draft and a sound velocity profile consisting of up to 10 pairs of depth and sound velocity. With the - UNIX is a trade mark of AT\&T Bell Labs. 
DECwriter terminal these parameters had to be entered sequentially at the keyboard, and typing errors meant having to start the sequence from the top. This fact becomes noteworthy when it is realized that the procedure must be followed not only when the system is first turned on or rebooted after a crash, but every time a new sound velocity profile must be entered to account for changing oceanographic conditions, as well as every few days to reset the Sea Beam clock which is known to drift about 20 seconds per day.

Once the parameter file in the VAX has been satisfactorily edited, the automated initialization method requires minimal user input, and the dialogue between the VAX and the Eclipse computers is displayed on the terminal and the printer for verification. With the terminal switch in the "Sea Beam" position the operator executes the start-up program for the Echo Processor: 'COMM'. Switching the terminal back to "VAX", the operator then executes the UNIX Cshell script 'RUN.loadsb' which sends the intialization parameters to the Eclipse computer. A shorter C-shell script 'RUNsvp.time' is used whenever it is only necessary to change the time or the sound velocity profile [9].

This automated procedure has been a great improvement by reducing the potential for operator error as well as the time required to change parameters during a survey hence minimizing data loss.

\section{Output Enhancements}

The Sea Beam system comes with a limited array of output devices and supporting features. Although it has the required driving circuitry to support 2 graphic recorders, 1 step-driven swath plotter, 1 analog storage oscilloscope, and 2 LED displays for depth readouts; only the storage scope and one LED display are standard equipment. The remaining devices must be purchased as optional equipment. On the R/V T. Washington, the output devices added in support of Sea Beam operations include a graphic recorder, two swath plotters and two belt-bed plotters.

The current graphic recorder is an EPC Labs model 3211 with 32 kilobytes of built-in memory. This graphic recorder is used to display the acoustic retum received either on the vertical beam, or on a selected port or starboard beam, or for one of the combinations of all port beams, all starboard beams or all beams port and starboard. If a second graphic recorder were added it would be limited to the vertical beam display only.

The memory feature of the graphic recorder plays an important role in the implementation of the Synch Box described in Section III, as it allows keying at non-specific times [8]. During Sync Box operations the graphic recorder is configured for use with an external record trigger. When Sea Beam is the only sound source in use, the recorder is set to its internal trigger mode and the EPC edge pulse is used for Sea Beam keying. Both the $3.5 \mathrm{kHz}$ subbottom profiler and the single channel seismic system use the same model of graphic recorders so that plenty of replacements and spares are available.

The Sea Beam system delivered to SIO in 1981 included one Houston Instrument Complot DP-1 plotter to display a near real-time swath of contoured Sea Beam data. This swath display includes the bottom contours, time, course, and contour interval. In addition, the rate of advance of the paper is a function of the ship's speed supplied to the Eclipse computer as described in Section II. This display is a very useful tool during the conduct of a survey as it provides the investigator with near real-time seafloor based navigation while following geological features of interest or positioning the ship prior to instrument and vehicle deployments as well as for coring and dredging operations. However, because this display does not contain any geographic information, to follow seafloor features often requires cutting and reorienting the strip of paper to match the current ship's heading. As this swath plot would be the only data available in case of failure or malfunction of the data logger, an archive copy is kept on microfilm. For this reason, a second Complot DP-1 plotter was connected in parallel to produce a continuous duplicate copy of the swath output, thereby granting the investigator unlimited shredding privileges on the working copy.

As mentioned above, plotting of the contoured Sea Beam data in a geographic reference frame requires that these data be merged with the ship's navigation. On the R/V T. Washington, this operation is performed by the VAX computer, and the data are plotted on one of the two CalComp 965A belt-bed plotters linked with the computer. There is no direct connection between the Sea Beam system and these plotters. During Sea Beam operations, one of the plotters is dedicated to plotting, with a few minutes delay, a swath of seafloor contours along the ship's track in a geographic reference frame. Parameters such as chart scale and contour interval are user selectable. The other plotter is used for Sea Beam chart making in the post-processing phase. This involves editing and processing the ship's navigation and remerging it with the Sea Beam data. Further information on the real-time and post-processing tasks associated with Sea Beam is found in Moore et al, [6] and Charters [10]. Also note that a similar plotting arrangement is used in the NECOR Sea Beam operation [7].

\section{Vertical Reference System}

Because the acoustic arrays of the Sea Beam system are mounted in a fixed position on the ship's hull, the system needs an accurate vertical reference to compensate for the roll and pitch motions of the ship. As mentioned in Section III, pitch compensation ensures that each of the 20 projector output pulses are properly phase shifted with respect to the center of the array, to align the maximum response axis of the transmitted acoustic radiation pattern with vertical. Likewise, the seafloor echoes received at the hydrophone array must be corrected for the ship's roll so that (1) the Narrow Beam Echo Sounder portion of the system can output the proper beams to the port, starboard, and vertical receivers and (2) the Echo Processor computer can compute the correct depth and cross track distance for each beam.

The Sea Beam system's specifications call for a vertical reference which outputs pitch and roll information in a synchro format with an accuracy of one tenth of one degree. Aboard the R/V $T$. Washington, one of three sensors can be selected for this task: (1) a vertical reference gyroscope (Kearfott-Singer) which was purchased with the Sea Beam system to serve as its source of vertical reference, (2) the gimbaled table of an Anschutz gravimeter and (3) the vertical reference unit of a Bell Aerospace BGM-3 gravimeter. A sensor is selected by connecting the appropriate jumpers in a gyro junction box.

The Kearfott-Singer gyroscope is the most "sea-worthy" vertical reference because it is not as adversely affected by rapid 180 course changes or rough sea-states $(>4)$ as the gravimeter references. It contains all the necessary electronics to provide the proper synchro output to the system. It requires $115 \mathrm{v}-400 \mathrm{~Hz}$ for its operation and it has intemal transformers which provide the necessary step down to $26 \mathrm{v}-400 \mathrm{~Hz}$ needed by the system for reference. Its only significant drawback is its limited mean-time between repairs: one year of constant use, after which the gyroscope's rotor bearings must be overhauled at the factory at a cost of $\$ 50,000$, with delivery times exceeding 6 months. For this reason this gyroscope is now only used as a back-up unit and in rough sea states. We do not plan to have this unit repaired the next time it fails.

As a substitute source of vertical reference, we used the gyrotable of an Anschutz gravimeter which was already installed on the R/V T. Washington. The gyrotable had two synchro devices giving 
pitch and roll information, so we only had to provide these synchros with the proper rotor input signals and the Sea Beam system with a 26v $400 \mathrm{~Hz}$ reference from an external transformer. This system worked well for several years but the need for frequent manual fine leveling of the gyrotable and constant maintenance due to its 20 years of age as well as the gyro's inability to re-erect itself quickly after drastic course changes were severe drawbacks. In February of 1987, the gravimeter system was replaced by a Bell Aerospace BGM-3 unit; however the gyrotable portion of the system still remains on board as an emergency vertical reference source.

The new gravimeter currently provides our primary source of vertical reference for Sea Beam. It is mounted in a small case on free moving gimbals aligned to true vertical by two independent gyroscopes, one for roll and one for pitch. The gravimeter was delivered with two resolvers mounted on the case to sense gimbal position. We built an interface to convert the output of these resolvers into the required synchro signals, and to provide the rotor input signal for the resolvers and a $26 \mathrm{v}-400 \mathrm{~Hz}$ reference signal for Sea Beam. At present this scheme is working well but the gyro devices used in the BGM-3 gravimeter also have a mean time between repairs of about 18 months with delivery schedules of 9 months and cost in excess of $\$ 10,000$.

In order to meet the vertical reference requirements of the Sea Beam system at a substantially lower cost, we are in the process of installing a Datawell PIRO-120 sensor on the R/ T. Washington. The interface between this sensor and the Sea Beam system uses a Z80 microprocessor and technology similar to that described in Section III for other components of the Sea Beam system designed by SCG.

An Input Buffer Amplifier for the Mechanical Roll Compensator

The Narrow Beam Echo Sounder portion of the Sea Beam system contains a mechanical roll compensator. This device couples a specified beam or combination of beams from the 16 preformed outputs of the beamformer to one or more of the port, starboard and vertical receivers for output to a graphic recorder and to a digitized vertical beam depth display. A roll servo system, consisting of an input synchro, a servo amplifier and a servo motor in a closed servo loop, takes roll synchro data from the vertical reference sensor. It rotates a shaft which moves the roll compensator plates to the correct position, thus coupling the proper beams to the receivers.

Due to the mechanical nature of the system, wear on the gear train and friction or binding of the compensator plates can cause a non zero servo error which is coupled back through the control transformer and loads the input signal. This can cause gross errors in the roll angle data as well as subtle errors which are difficult to detect. In addition, the roll input control transformer is highly inductive and has a low input impedance, resulting in a significant phase shift (up to $20^{\circ}$ ) between the reference and output windings depending on the the vertical reference sensor used.

This problem required careful attention because roll data are also used by the Eclipse computer to compensate for the ship's roll while calculating depths and horizontal distances. Given that the output synchros could not be changed, our first modification was to provide a phase shift $(R C)$ network which matched the reference phase to that of the output. Although functional, this method proved impractical because of the need to reconfigure the input wiring whenever a different vertical reference sensor was used, and subtle roll errors could still happen if any loading occurred.

A buffer amplifier circuit, installed between the roll angle input and the roll compensator synchro, provided a better fix to the problem. This prevents any synchro loading from affecting the input and allows us to use any of our vertical references without the need for any external phase shift network or rewiring. The circuit consists of three high power operational amplifiers, one amplifier for each winding S1, S2, and S3. The amplifiers are wired as voltage followers so that the input voltage is equal to the output voltage and is in phase for each respective winding. Sufficient voltage sources $(+,-24 \mathrm{Vdc})$ are provided to insure linearity over the entire input range, approximately $22 \mathrm{Vp}$-p. Output filter capacitors are also included to prevent spurious noise from affecting signal accuracy.

This modification has proved very effective in assuring the accuracy of the input roll signal and the ease of switching between any of our vertical reference sources.

\section{Acoustic Data Acquisition}

The ability to make, for every transmission cycles, 16 discrete acoustic measurements with an angular resolution of roughly $22 / 3^{\circ}$ within an angular sector of about $40^{\circ}$ centered on the ship's vertical axis is the basis for the distinct advantage of a multibeam echosounder such as Sea Beam over a single point depth sounder, whether wide or narrow beam. Such simultaneous acoustic measurements contain much more information than is necessary for bathymetry. However, there is no internal provision in the Sea Beam system to preserve the acoustic information received, and the signals once processed for bathymetry are discarded. In 1981, when the Sea Beam system was installed on the R/V T. Washington, the Marine Physical Laboratory (MPL) at SIO explored ways to preserve this acoustic information. A set of buffer amplifiers were built to tap the detected and rectified envelopes of the beamformed echo signals received on the 16 beams. These signals were sent differentially to a special purpose acoustic data acquisition system where they were digitized and recorded on magnetic tape for further processing [10].

Acoustic data recorded with this system have proved invaluable to assess the performance of the Sea Beam system and to explain the cause of artifacts that were sometimes found in the contoured bathymetry output by the system [3]. The addition of buffer amplifiers at the output of the receivers in the Echo Processor also highlighted a $1 \mathrm{MHz}$ noise endemic to the Echo Processor. This problem was successfully alleviated by adding capacitors to the buffer amplifiers. Since then, GIC has released a Sea Beam application note advising the addition of a capacitor on the backplane of the Echo Processor receivers. Duplicates of the buffer amplifier boards were also made at MPL and given to the NECOR Sea Beam group who installed similar acoustic data acquisition capabilities on the R/N Conrad and Atlantis II.

For seafloor acoustic backscattering investigations, the acoustic data recorded in this fashion proved only partly adequate as sidelobe interference inherent in the multibeam geometry affect the returns. This situation is particularly damaging in the near-specular beams were sidelobe interference and bottom retum overlap. As only the envelope of the returns was recorded, there was no way to separate sidelobe interference from bottom retum; to do so requires that the full waveform be available [11].

For this reason, in 1985, the MPL data acquisition system was redesigned to allow preservation of the amplitude and the phase of the echoes received. This is done by tapping the signals at the output of the beamformer, where they are still in audio form, and by basebanding and quadrature sampling to obtain the in-phase (I) and quadrature $(Q)$ components of these signals. The resulting 32 channels ( 16 complex channels) are then digitized at approximately $1 \mathrm{kHz}$ per channel, and recorded on magnetic tape. This complex acoustic data acquisition scheme, described in greater detail by de Moustier and Pavlicek [12], has been used successfully aboard the R/V's $T$. Washington, Atlantis II and the French Oceanographic Vessel Jean Charcot. Complex acoustic data derived from this system have also confirmed the fact that Adaptive Noise Cancelling technique were well suited to remove the sidelobe interference and give access to 
seafloor acoustic backscatter measurements with the anticipated angular resolution of roughly $22 / 3^{\circ}[13]$.

\section{$\checkmark$ CONCLUSIONS}

In spite of the problems encountered during the first two years of operation of the Sea Beam system, which led to the modifications described in this paper, the system has proved very reliable. This fact is a tribute to the designers of the system as most of the Narrow Beam Echo Sounder sub-system delivered to SIO had remained unchanged since it first came out in the mid 1960's. The modifications made to the system installed on the R/V T. Washington have reduced the need for maintenance or repairs as well as facilitated the task of the operator. Although these modifications are specific to the SIO system, the automated parameter intialization, the multiple sound source synchronization, the buffering of the roll input and the improvements in calibration procedures can be adapted to other Sea Beam installations that include a data logging computer.

The modifications described in this paper remain relatively benign as they were intended to improve the existing system rather than design a new one. By today's standards [14] the Sea Beam system will need to undergo more drastic changes to meet the needs of the oceanographic community for wider swath widths and finer angular resolution. This will require modification of the acoustic arrays and the use of digital beamforming techniques.

\section{ACKNOWLEDGEMENTS}

The development work reported in this paper has been funded in part under the Accelerated Research Initiative on multibeam systems sponsored by the Office of Naval Research (ONR), Contract N00014-86-G-0142, N00014-85-G-0104 (SCG) and N00014-79-C0472 (MPL); and from SIO Institutional funds. The Sea Beam system installed on the R/V T. Washington was purchased with an ONR grant, Contract NOOO14-80-C-0440, matched equally with trust funds from SIO. J. L. Abbott directed and was actively involved in the development work done by the Shipboard Computer Group. Under his guidance the main contributors to the hardware development were P. Downes, T. Hylas and D. Stuber for the STD data acquisition system, P. Downes for the digital pitch compensator, J.C. Phillips, T. Hylas and D. Stuber for the Synch Box, and T. Hylas for all the other modifications to the Sea Beam system. The Sea Beam acoustic data acquisition work done at MPL was initiated by R.C. Tyce and pursued by C. de Moustier and F.V. Pavlicek who have developed the necessary hardware and software, with significant software contributions by R. M. Lawhead. We thank E. Ford for typing and formatting and J. Griffith for the art work.

\section{REFERENCES}

[1] Renard, V. and J. P.Allenou, Sea Beam multi-beam echo-sounding in "Jean Charcot" Description, evaluation and first results International Hydrographic Review, LVI(1), pp. 35-67, 1979.

[2] Farr, H. K., Multibeam bathymetric sonar: Sea Beam and Hydrochart, Marine Geodesy, 4(2), pp. 77-93, 1980.

[3] de Moustier, C. and M. C. Kleinrock, "Bathymetric antifacts in Sea Beam data: how to recognize them, what causes them", J. Geophys. Res., Vol 91, No B3, pp. 3407-3424, 1986.

[4] Abbott J.L., S.M. Smith, J.S. Charters, P.G. Downes, T. Hylas, R.L. Moe, J.M. Moore and D.V. Stuber, Scripps seagoing computer centers: real-time data acquisition and processing, IEEE Proc. 4th Working Symposium on Oceanographic Data Systems, pp. 123-129, 1986.

[5] Charters J.S., SIO Sea Beam software documentation, Shipboard Computer Group A-023, Scripps Inst. of Oceanog., La Jolla CA 92093. unpublished document, 1986.

[6] Moore J. M., J. S. Charters and C. de Moustier, Multi-sensor realtime data acquisition and preprocessing at sea, Proc. MTS-IEEE Oceans' 88 Conf., Baltimore Maryland, 1988.

[7] Tyce R.C., S. Ferguson and P. Lemmond, NECOR Sea Beam data collection and processing development, MTS Joum., Vol. 21 No. 2, pp. 80-92, 1987.

[8] Phillips J.C., J.L. Abbott and C. de Moustier, Multiple sound source synchronizer for seafloor surveying, OTC \#5867, Proc. Offshore Technology Conference, Houston Texas, 1988.

[9] Smith S.M., J.L. Abbott and J.C. Phillips, Scripps Sea Beam start up procedure and downloading of terminal entry parameters, SIO Geol. Data Center, Sea Beam Series No. 5, internal unpublished report, 1987.

[10] de Moustier, C., Sea Beam Acoustic Data Acquisition System, Technical Memorandum 379, Scripps Institution of Oceanography, San Diego, CA, 1985

[11] de Moustier, C. "Beyond bathymetry: mapping acoustic backscattering from the deep seafloor with Sea Beam", J. Acoust. Soc. Am., Vol 79, No 2, pp. 316-331, 1986.

[12] de Moustier C. and F.V. Pavlicek, A fully transportable Sea Beam complex acoustic data acquisition system, OTC \#5514, Proc. Offshore Technology Conf., Houston Texas, pp. 269-274, 1987.

[13] Alexandrou D. and C. de Moustier, Adaptive noise cancelling applied to Sea Beam sidelobe interference rejection, IEEE J. of Oceanic Eng., Vol. 13 No. 2, pp. 70-76, 1988.

[14] de Moustier C., State of the art in swath bathymetry survey systems, in Current Practices and New Technology in Ocean Engineering, G.K. Wolfe and P.Y. Chang Eds, ASME OED-Vol. 13, pp. 29-38, 1988. 